\title{
LMO3 promotes gastric cancer cell invasion and proliferation through Akt-mTOR and Akt-GSK3ß signaling
}

\author{
YAN-SONG QIU $^{1}$, NING-NING JIANG ${ }^{1}$, YAN ZHOU ${ }^{1}$, KUAI-YUN YU ${ }^{1}$, \\ HONG-YAN GONG ${ }^{1}$ and GUANG-JUN LIAO ${ }^{2}$
}

Departments of ${ }^{1}$ General Surgery and ${ }^{2}$ Bone Tumors, Yantai Mountain Hospital, Yantai, Shandong 264000, P.R. China

Received June 12, 2017; Accepted January 31, 2018

DOI: $10.3892 /$ ijmm.2018.3476

\begin{abstract}
The present study assessed the biological functions of LIM domain only 3 (LMO3) in gastric cancer (GC) investigated and the underlying molecular mechanisms. It was revealed that the expression of LMO3 was significantly upregulated in GC tissues. A GC tissue microarray $(n=164)$ indicated that LMO3 expression was closely associated with clinicopathological factors, as well as overall survival and disease-free survival of patients. After knockdown of LMO3 in MGC-803 and SGC-7901 cells, the invasion and proliferation were obviously suppressed. Furthermore, LMO3 knockdown suppressed the phosphorylation of Akt, mammalian target of rapamycin (mTOR) and glycogen synthase kinase (GSK) $3 \beta$ signaling. An inhibitor of mTOR, dactolisib, abrogated recombinant LMO3 protein-induced GC cell invasion and proliferation, while an inhibitor of GSK3 $\beta$, CHIR-98014, only abrogated rLMO3 protein-induced proliferation. These results suggested that $\mathrm{LMO} 3$ promotes $\mathrm{GC}$ cell invasion and proliferation mainly through Akt/mTOR and Akt/GSK3 $\beta$ signaling. LMO3 may serve as a potential therapeutic target for $\mathrm{GC}$ in the future.
\end{abstract}

\section{Introduction}

Gastric cancer (GC) is the second most common type of malignancy worldwide. In developed western countries, the 5 -year survival rate for GC is as low as $10-19 \%$ (1). In China, $\mathrm{GC}$ is the most common type of malignancy according to the number of cases diagnosed annually (2). It is currently elusive what factors contribute to the development, progression and metastasis of GC in geographic areas with highly prevalence, including certain cities in Northwest China $(3,4)$. GC includes

Correspondence to: Dr Guang-Jun Liao, Department of Bone Tumors, Yantai Mountain Hospital, 91 Jiefang Road, Zhifu, Yantai, Shandong 264000, P.R. China

E-mail: gjliao@yeah.net

Key words: LIM domain only 3, gastric cancer, invasion, proliferation, Akt, mammalian target of rapamycin, glycogen synthase kinase $3 \beta$ two distinct morphological subtypes: Gastric intestinal type adenocarcinoma and diffuse gastric adenocarcinoma (5-7). In addition to tumor protein 53 mutations, studies have reported frequent inactivating mutations in genes associated with cell adhesion and chromatin remodeling $(8,9)$. Although certain essential factors that may serve as therapeutic tagets were identified in recent years, effective methods to treat GC or monitor disease progression in the clinic are still lacking (10-13).

The LIM domain only (LMO) protein family contains four members: LMO1, LMO2, LMO3 and LMO4. Although LMO proteins lack DNA-binding activity, they were reported to be involved in the transcriptional regulation of specific target genes in collaboration with other transcription factors (2). Genetic analyses demonstrated that LMO1 and LMO2 contribute to the genesis of immature and aggressive T-cell leukemia (14), while LMO4 has been reported to be implicated in the development of breast cancer $(15,16)$. LMO3 forms a complex with the neuronal-specific basic helix-loop-helix (HLH) transcription factor, HLH protein 2, which is also overexpressed in neuroblastoma with unfavorable prognosis compared with that in neuroblastoma with favorable prognosis. Furthermore, LMO3 has been reported to have important roles in numerous cancer types, including neuroblastoma $(17,18)$ and lung cancer $(19,20)$.

The present study reported that LMO3 expression was significantly upregulated in GC tissues and was closely associated with the clinicopathological characteristics and patient prognosis. Knockdown of LMO3 significantly suppressed the invasion and proliferation of GC cells. Furthermore, the effects of LMO3 on the invasion and proliferation of GC cells were determined to be dependent on Akt/mammalian target of rapamycin (mTOR) and Akt/glycogen synthase kinase (GSK) $3 \beta$ signaling.

\section{Materials and methods}

Cell culture. Human GC cell lines, including AGS, BGC-823, HGC-27, MGC-803, MKN-45 and SGC-7901, were purchased from the Cell Bank of the Chinese Academy of Sciences (Shanghai, China). BGC-823, MGC-803, MKN-45 and SGC-7901 cells were cultured in RPMI-1640 medium (Gibco, Thermo Fisher Scientific, Inc., Waltham, MA, USA) supplemented with $10 \%$ (v/v) fetal calf serum 
(FCS) (Gibco, Thermo Fisher Scientific, Inc.) and 1\% antibiotics. AGS was cultured in DMEM F-12 medium (Gibco, Thermo Fisher Scientific, Inc.) supplemented with 10\% FCS and $1 \%$ antibiotics. HGC-27 was cultured in RPMI-1640 medium supplemented with $20 \%$ FCS and $1 \%$ antibiotics. All of the $\mathrm{GC}$ cells were cultured at $37^{\circ} \mathrm{C}$ in a humidified atmosphere containing $5 \% \mathrm{CO}_{2}$.

Clinical samples. Human gastric tumor $(\mathrm{n}=19)$ and normal tissues ( $\mathrm{n}=15)$, in 10 cases of which gastric tumor and normal tissues were paired, were obtained from patients who were admitted between January 2012 and December 2016 at the Department of Bone Tumor (collected form the Department of General Surgery, at which the surgery was performed), Yantai Mountain Hospital (Yantai, China). All of the patients provided written informed consent prior to enrollment, and the study was approved by the Research Ethics Committee of Yantai Mountain Hospital (Yantai, China). In addition, a human tissue microarray, which was also obtained from the Department of Bone Tumor (collected form the Department of General Surgery), Yantai Mountain Hospital (Yantai, China) was used, which contained GC samples and paired normal tissues from 164 cases.

KMplot analysis. The Affy ID of the dataset used in the presnet study was 204424_s_at. The best cut-off value was auto selected using KMplot (http://kmplot.com/analysis/ index.php?p=service\&cancer=gastric).

Reverse transcription-quantitative polymerase chain reaction $(R T-q P C R)$. Total RNA was extracted using TRIzol (Takara Bio Inc., Otsu, Japan) and reverse transcribed with a PrimeScript RT-PCR kit (Perfect Real-Time; Clontech; Takara Bio Inc.). Real-time qPCR analyses were performed with SYBR Premix Ex Taq (Takara Bio Inc.) on a 7500 Real-Time PCR System (Applied Biosystems; Thermo Fisher Scientific, Inc., Waltham, MA, USA) at the recommended thermal cycling settings: One initial cycle at $95^{\circ} \mathrm{C}$ for $30 \mathrm{sec}$, followed by 40 cycles of $5 \mathrm{sec}$ at $95^{\circ} \mathrm{C}$ and $31 \mathrm{sec}$ at $60^{\circ} \mathrm{C}$. Results were quantified using the $2^{-\Delta \Delta C q}$ method was used (21). The primer sequences used were as follows: LMO3 forward, 5'-TCTGAGGCTCTTTGGTGT AACG-3' and reverse, 5'-CCAGGTGGTAAACATTGTCCT TG-3'; and $\beta$-actin forward, 5'-CTCCATCCTGGCCTCGCT GT-3' and reverse, 5'-GCTGTCACCTTCACCGTTCC-3'.

Western blot analysis. Cells were lysed in lysis buffer (50 mM Tris- $\mathrm{HCl}, 150 \mathrm{mM} \mathrm{NaCl}, 1 \%$ Triton X-100, $1 \mathrm{mM}$ each $\mathrm{MgCl}_{2}, \mathrm{MnCl}_{2}$ and $\mathrm{CaCl}_{2}, 1 \mathrm{mM}$ phenylmethylsulfonyl fluoride and $10 \mathrm{mM}$ sodium fluoride; Sangon, Shanghai, China). The protein concentration was determined using the BCA method. A total of $20 \mu \mathrm{g}$ protein was loaded per lane and proteins were separated by 6-12\% SDS-PAGE under reducing conditions and transferred onto a nitrocellulose membrane (Thermo Fisher Scientific, Inc.). The membrane was blocked in PBS/Tween-20 containing 5\% bovine serum albumin (Sangon Biotech Co., Ltd., Shanghai, China), followed by incubation with the antibodies for LMO3 (1:1,000, cat. no. ab69573; Abcam, Cambridge, MA, USA), phospho-Akt $(1: 2,000$, cat. no. 4060), total-Akt $(1: 2,000$, cat. no. 4691), phospho-mTOR (1:2,000, cat. no. 5536), total-mTOR (1:2,000, cat. no. 2983), phospho-GSK3 $\beta$ (1:2,000, cat. no. 5558), total-GSK3 $\beta(1: 2,000$, cat. no. 12456; all from Cell Signaling Technology, Inc., Danvers, MA, USA) and GAPDH (1:1,000, cat. no. 9001-50-7; Sigma-Aldrich; Merck KGaA, Darmstadt, Germany) at $4^{\circ} \mathrm{C}$ overnight. Subsequently, the membrane was washed and incubated with IRDye ${ }^{\circledR}$ 680LT Goat anti-Rabbit $\operatorname{IgG}\left(\mathrm{H}+\mathrm{L}, 1: 10,000\right.$, cat. no. P/N 925-68021) or IRDye ${ }^{\circledR}$ 800CW Goat anti-Mouse IgG (H+L, 1:10,000, cat. no. P/N 925-32210; LI-COR Biosciences, Lincoln, NE, USA) at room temperature for $1 \mathrm{~h}$. The fluorescently-labeled secondary antibodies were directly detected with the Odyssey imaging system (LI-COR Biosciences).

Small interfering (si)RNA transfection. siRNA duplexes for LMO3 were produced by Genepharma (Shanghai, China). The LMO3 siRNA and scrambled siRNA were purchased from Abnova (cat. no. H00055885-R01; Abnova, Taiwan, China). Transfection steps were performed according to the manufacturer's protocols of the X-tremeGENE siRNA transfection reagent (cat. no. 4476093001; Sigma-Aldrich; Merck KGaA).

Recombinant (r)LMO3 protein and inhibitors. $\mathrm{LLMO} 3$ protein was purchased from Abnova (Taipei, Taiwan). The inhibitor of mTOR (dactolisib) and the inhibitor of GSK3 $\beta$ (CHIR-98014) were purchased from Selleck Chemicals (Houston, TX, USA). rLMO3 protein was added to BGC-823 and HGC-27 cells, and dactolisib or CHIR-98014 were added to above cells $2 \mathrm{~h}$ later. All cells were incubated at $37^{\circ} \mathrm{C}$.

Invasion assay. MGC-803, SGC-7901, BGC-823 or HGC-27 cells were detached, resuspended in serum-free RPMI-1640 medium, and seeded at $2 \times 10^{4}$ cells per well in $100 \mu \mathrm{l}$ in 8- $\mu \mathrm{m}$ Transwell inserts (EMD Millipore, Billerica, MA, USA) with membranes coated in Matrigel (BD Biosciences, Franklin Lakes, NJ, USA) on top of a 24-well plate. RPMI-1640 medium containing 5\% FBS was added to the bottom chamber. Cells were incubated at $37^{\circ} \mathrm{C}$ for $48 \mathrm{~h}$. Subsequently, filters were fixed and stained with $0.1 \%(\mathrm{w} / \mathrm{v})$ crystal violet at room temperature for $20 \mathrm{~min}$. Non-invading cells were removed from the upper side of the membrane and invaded cells on the lower side were counted under a microscope at a magnification of $x 400$. At least three grids (each grid was restricted at a field $\sim 982 \times 722 \mathrm{~mm}$, with a magnification of $\times 400$ ) per field were counted and the experiments were repeated twice.

Cell viability assay. Cell viability was determined using a standard Cell Counting Kit-8 (CKK-8) assay. MGC-803, SGC-7901, BGC-823 or HGC-27 cells were seeded into 96-well plates $\left(100 \mu 1\right.$ per well) at a density of $2 \times 10^{4}$ cells per $\mathrm{ml}$, and the total number of cells seeded in each well was $2 \times 10^{3}$. MGC-803 and SGC-7901 cells were transfected with LMO3 siRNA or scrambled siRNA before these cells were seeded. However, BGC-823 and HGC-27 cells were treated with rLMO3 protein, dactolisib or CHIR-98014 after the cells adhered to the bottom of dishes. After incubation for at 12, 24, 48 and 72 h, $10 \mu$ l CKK-8 reagent (Dojindo, Kumamoto, Japan) was added to each well. After $2 \mathrm{~h}$ of incubation at $37^{\circ} \mathrm{C}$, the optical density was measured using a microplate reader at a wavelength of $450 \mathrm{~nm}$. 

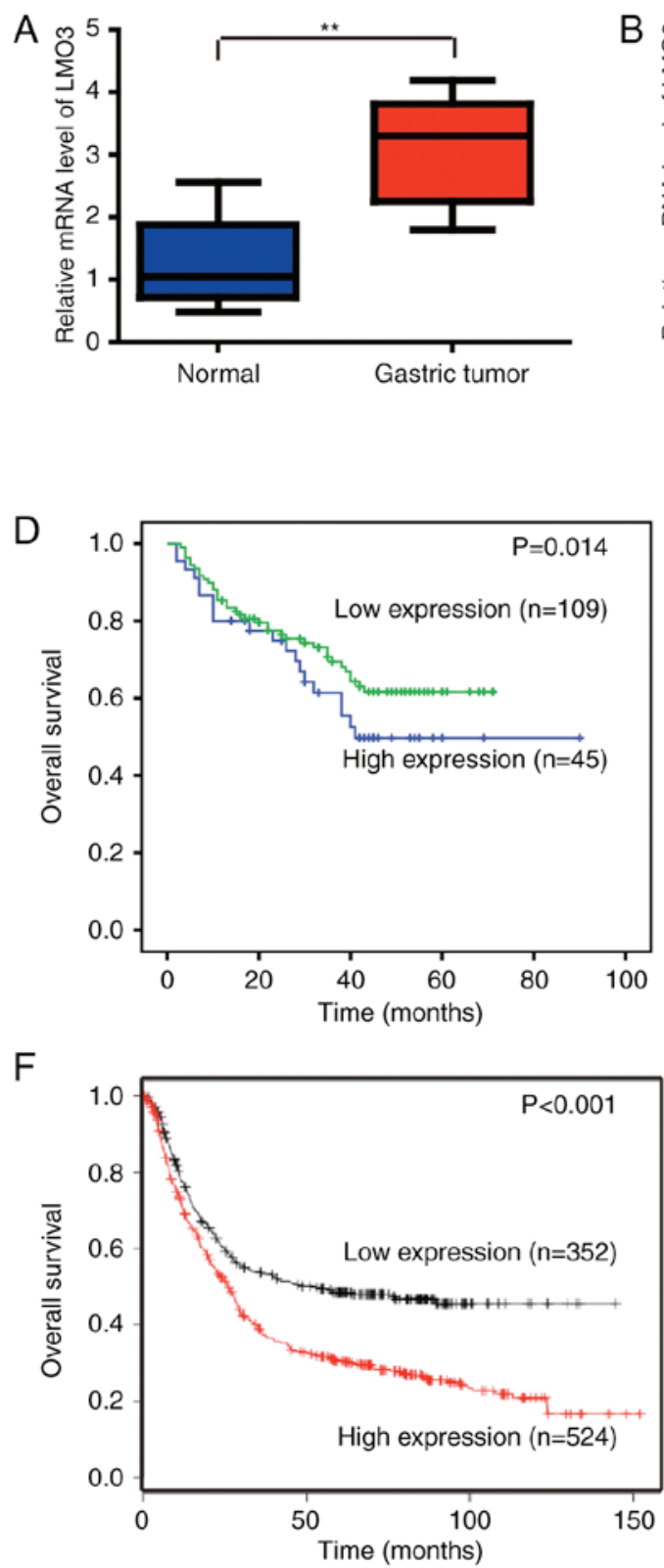
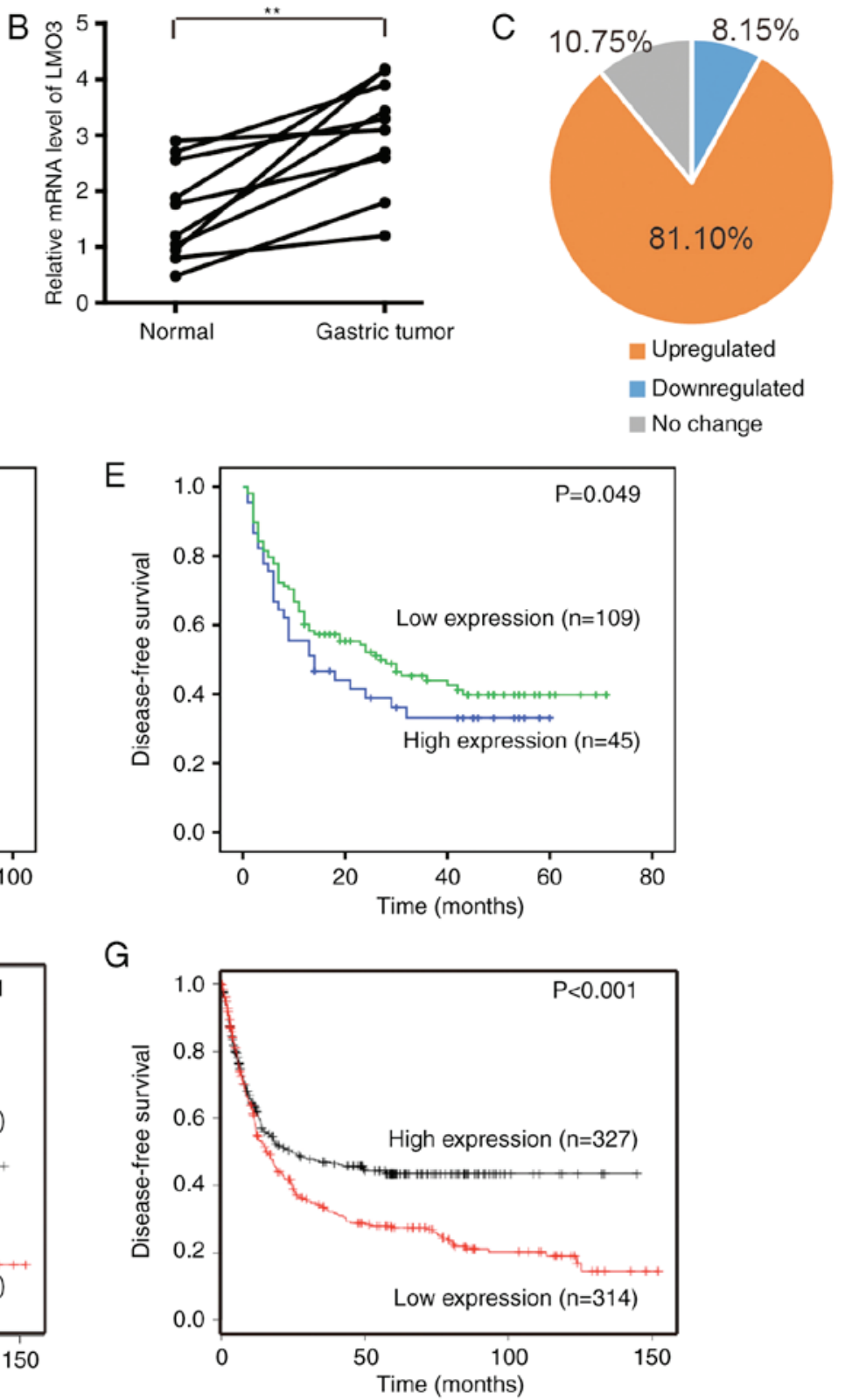

Figure 1. LMO3 expression is upregulated in GC and closely associated with patient prognoses. mRNA expression levels of LMO3 in (A) 19 gastric tumor (median=1.05, 0.48 to 2.56) and 15 normal gastric tissues (median=3.30, 1.80 to 4.19); and (B) 10 paired gastric tumor (1.629 \pm 0.273 ) and normal tissues (3.038 \pm 0.312$)$ within this cohort. ${ }^{* *} \mathrm{P}<0.01$. (C) A GC tissue microarray $(\mathrm{n}=164)$ indicated that the expression of LMO3 was upregulated in $81.10 \%$ of GC tissues. (D and E) Kaplan-Meier analysis of (D) OS ( $\mathrm{P}=0.014)$ and (E) DFS $(\mathrm{P}=0.049)$ for GC patients from the microarray stratified by the expression of LMO3. Scoring of immunohistochemical staining was conducted according to the ratio and intensity of positive-staining cells: 0-35\% was designated as a low expression group; $>36 \%$ was designated as a high expression group. (F and G) Kaplan-Meier plot dataset analysis using the KMplot online tool to determine (F) OS (P<0.001) and (G) DFS (P<0.001) of GC patients stratified by the expression of LMO3 (Affy ID of the dataset, 204424_s_at). The best cutoff value was auto selected using KMplot. DFS, disease-free survival; OS, overall survival; GC, gastric cancer; LMO3, LIM domain only 3.

Statistical analysis. Values are expressed as the mean \pm standard error of the mean. Statistical analyses were performed using SPSS 16.0 for windows (SPSS, Inc., Chicago, IL, USA). Survival time was analyzed with the Kaplan-Meiermethod.Theassociation between LMO3 expression and the clinicopathological features of patients with gastric cancer was evaluated using Pearson's Chi-square test. One-way analysis of variance was used for comparison between groups. Multiple comparison between the groups was performed using Student-Newman-Keuls post hoc method. $\mathrm{P}<0.05$ was considered to indicate a statistically significant difference.

\section{Results}

LMO3 expression is closely associated with clinicopathological characteristics and prognosis of GC patients. To investigate the expression of LMO3 in GC tissues, 19 gastric tumor and 15 normal gastric tissues were collected and analyzed by RT-qPCR. The expression levels of LMO3 were significantly upregulated in GC tissues (Fig. 1A). In 10 paired gastric tumor and normal tissues within this cohort, LMO3 expression was also upregulated in GC tissues (Fig. 1B). 
A GC tissue microarray $(n=164)$ was then used to investigate the correlation between LMO3 expression and clinicopathological characteristics and patient prognoses. Scoring of immunohistochemical staining was conducted according to the ratio and intensity of positive-staining cells: $0-35 \%$ was designated as the low expression group; $>36 \%$ was designated as the high expression group. The age of 62 years, the median age of this patient cohort, was set as a cut-off, based on which the patients were stratified into two groups. Notably, no significant difference was indicated among the age groups. It was identified that the expression of LMO3 was upregulated in $81.10 \%$ of GC tissues (Fig. 1C). Furthermore, the expression of LMO3 was closely associated with the tumor size, differentiation, vascular embolism and tumor-nodes-metastasis (TNM) stage (Table I), and high expression of LMO3 was positively correlated with poor overall survival (OS; $\mathrm{P}=0.014)$ or disease-free survival (DFS; P=0.049; Fig. 1D and E, respectively). The above results were confirmed by analyzing the datasets (Affy ID 204424_s_at) with the software Kaplan Meier-plotter (http://kmplot.com/analysis/index. $\mathrm{php}$ ? $\mathrm{p}=$ service $\&$ cancer $=$ gastric), revealing that LMO3 expression was positively correlated with poor OS $(\mathrm{P}<0.001)$ and DFS $(\mathrm{P}<0.001)$ of $\mathrm{GC}$ patients (Fig. $1 \mathrm{~F}$ and $\mathrm{G})$.

Knockdown of LMO3 in MGC-803 and SGC-7901 cells. To further investigate the biological functions of LMO3 in GC, the expression level of LMO3 were first detected in $6 \mathrm{GC}$ cell lines. As presented in Fig. 2A, LMO3 was highly expressed in MGC-803 and SGC-7901 cells compared with the other cell lines. Therefore, the MGC-803 and SGC-7901 cell lines were subjected to knockdown of LMO3 by using siRNA (labeled as si-LMO3-1 and si-LMO3-2). Through RT-qPCR and western blot analysis, it was demonstrated that LMO3 was successfully silenced in MGC-803 and SGC-7901 cells (Fig. 2B-E).

LMO3 knockdown suppresses the invasion and proliferation of GC cells. The present study investigated the role of LMO3 in the invasion of GC cells. A Transwell Matrigel invasion assay demonstrated that knockdown of LMO3 suppressed the invasive capacity of MGC-803 (Fig. 3A and B) and SGC-7901 (Fig. 3C and D) cells after $48 \mathrm{~h}$.

Furthermore, a CCK-8 cell viability assay was employed to investigate the role of LMO3 in the proliferation of GC cells. It was revealed that the viability of MGC-803 and SGC-7901 cells was significantly suppressed by knockdown of LMO3 at the 24, 48 and $72 \mathrm{~h}$ time-points (Fig. 3E and F).

LMO3 knockdown suppresses Akt, mTOR and GSK3 $\beta$ signaling in $G C$ cells. To investigate the underlying mechanism of the role of LMO3 in GC, the Akt/mTOR and Akt/GSK3 $\beta$ signaling pathways were assessed in MGC-803 cells by western blot analysis. Of note, LMO3 knockdown significantly suppressed the phosphorylation of Akt (Fig. 4A and B). The phosphorylation of mTOR, a downstream signaling event of Akt, was also suppressed by silencing LMO3 (Fig. 4C and D). Furthermore, the phosphorylation of GSK3 $\beta$, another downstream signaling event of Akt, was also suppressed by silencing LMO3 (Fig. 4E and F).
Table I. Correlation of clinicopathological factors with LMO3 expression.

\begin{tabular}{|c|c|c|c|}
\hline \multirow[b]{2}{*}{ Variable } & \multicolumn{2}{|c|}{ LMO3 } & \multirow[b]{2}{*}{ P-value } \\
\hline & High (n) (\%) & Low (n) $(\%)$ & \\
\hline Age (years) & & & 0.734 \\
\hline$\leq 62$ & $24(14.63)$ & $53(32.32)$ & \\
\hline$>62$ & $25(15.24)$ & $62(37.80)$ & \\
\hline Gender & & & 0.668 \\
\hline Female & $6(3.66)$ & $17(10.37)$ & \\
\hline Male & $43(26.22)$ & $98(59.76)$ & \\
\hline Smoking history & & & 0.276 \\
\hline Yes & $37(22.56)$ & 77 (46.95) & \\
\hline No & $12(7.32)$ & $38(23.17)$ & \\
\hline Lauren subtype & & & 0.787 \\
\hline Diffuse & $23(14.11)$ & $61(37.42)$ & \\
\hline Intestine & $25(15.34)$ & $54(33.13)$ & \\
\hline Location & & & 0.447 \\
\hline Upper stomach & $11(6.71)$ & $20(12.20)$ & \\
\hline Middle stomach & $22(13.41)$ & $65(39.63)$ & \\
\hline Lower stomach & $13(7.93)$ & $24(14.63)$ & \\
\hline Remnant stomach & $3(1.83)$ & $6(3.66)$ & \\
\hline Tumor size (cm) & & & $<0.001^{\mathrm{a}}$ \\
\hline$\leq 5$ & $17(10.37)$ & $78(47.56)$ & \\
\hline$>5$ & $32(19.51)$ & $37(22.56)$ & \\
\hline Differentiation & & & $0.004^{\mathrm{a}}$ \\
\hline Well & $1(0.61)$ & $2(1.23)$ & \\
\hline Moderate & $11(6.75)$ & $59(36.20)$ & \\
\hline Poor & $36(22.09)$ & $54(33.13)$ & \\
\hline Vascular embolism & & & $0.001^{\mathrm{a}}$ \\
\hline Yes & $35(21.34)$ & $105(64.02)$ & \\
\hline No & $14(8.54)$ & $10(6.10)$ & \\
\hline TNM stage & & & $0.004^{\mathrm{a}}$ \\
\hline I & $30(18.40)$ & $87(53.37)$ & \\
\hline II & $4(2.45)$ & $18(11.04)$ & \\
\hline $\mathrm{III} / \mathrm{IV}$ & $14(8.59)$ & $10(6.13)$ & \\
\hline
\end{tabular}

${ }^{\text {a }}<<0.05$. Pearson's $\chi^{2}$ test was used for statistical analysis. TNM, tumor-nodes-metastasis; LMO3, LIM domain only 3. Scoring of immunohistochemical staining was conducted according to the ratio and intensity of positive-staining cells: $0-35 \%$ was designated as a low expression group; $>36 \%$ was designated as a high expression group.

Taken together, the abovementioned results suggested that LMO3 promotes the invasion and proliferation of GC cells through Akt/mTOR and Akt/GSK3 $\beta$ signaling.

LMO3 promotes GCcell invasion through Akt/mTOR signaling, and supports GC cell proliferation via Akt/mTOR and Akt/GSK3 $\beta$ signaling. Since mTOR and GSK $3 \beta$ are different downstream signaling events of Akt, the effects of LMO3 on 


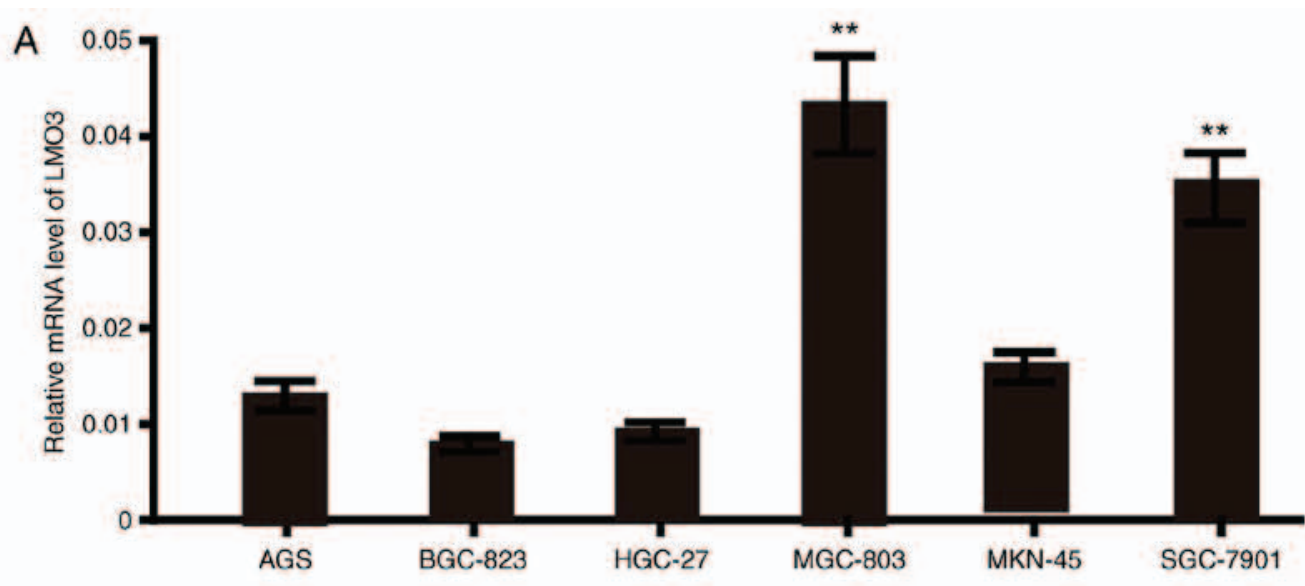

B

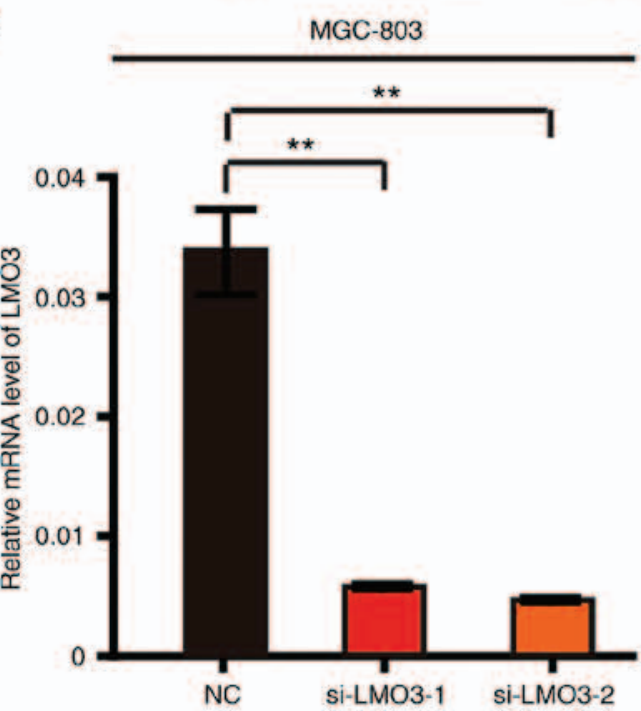

C

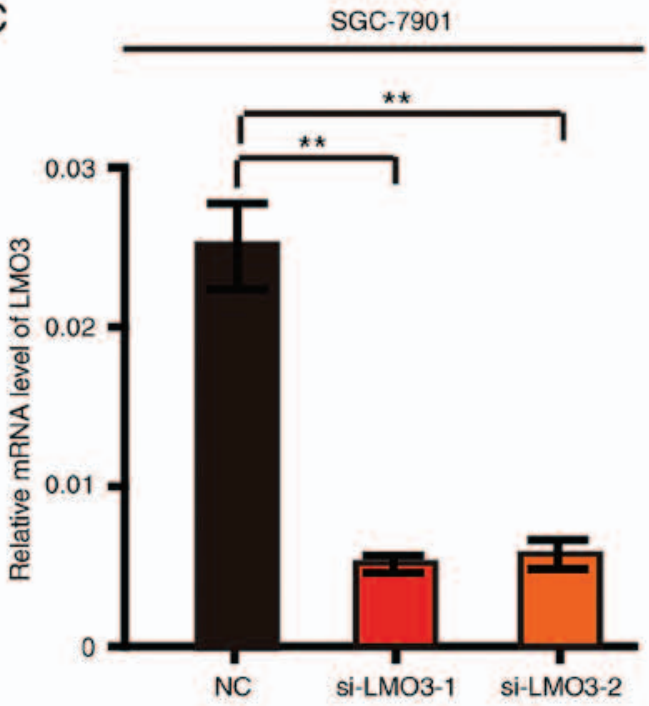

D

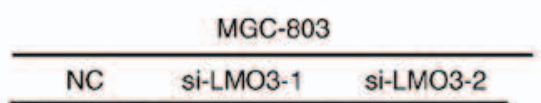

$E$
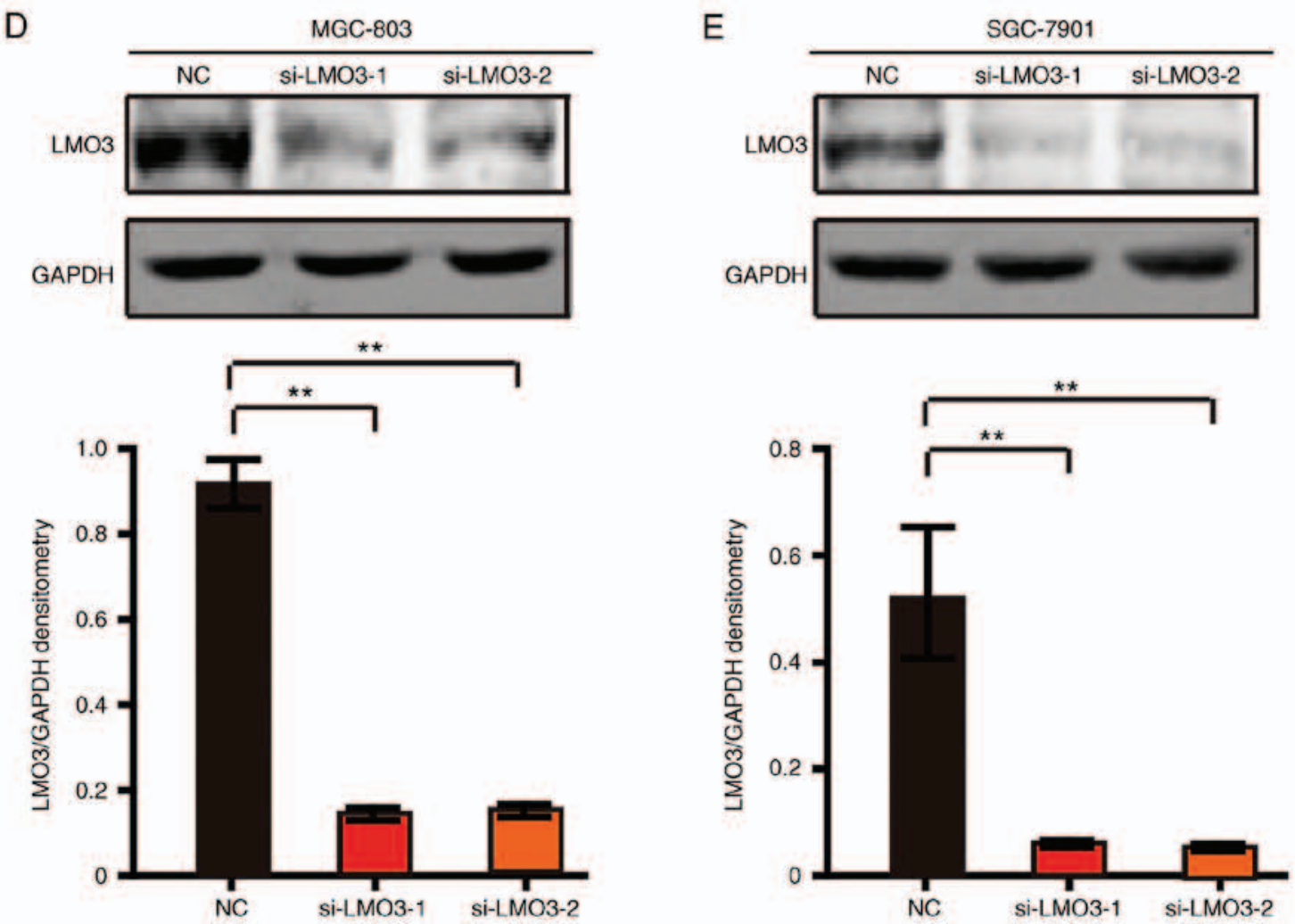

Figure 2. Expression of LMO3 in GC cell lines and knockdown of LMO3. (A) Expression of LMO3 in the GC cell lines AGS, BGC-823, HGC-27, MGC-803, MKN-45 and SGC-7901. (B and C) mRNA expression levels of LMO3 in (B) MGC-803 and (C) SGC-7901 cells following transfection with siRNA targeting LMO3. (D and E) Protein expression levels of LMO3 in (D) MGC-803 and (E) SGC-7901 cells following transfection with siRNA targeting LMO3. "* P<0.01. GC, gastric cancer; LMO3, LIM domain only 3; NC, negative control; siRNA, small interfering RNA. 
A

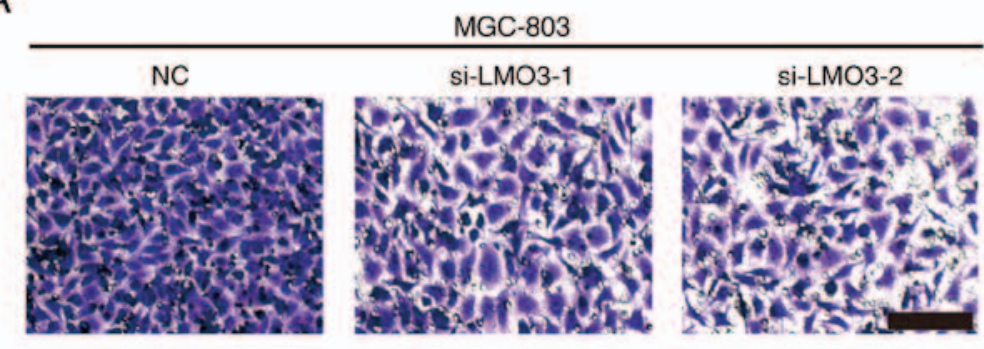

C

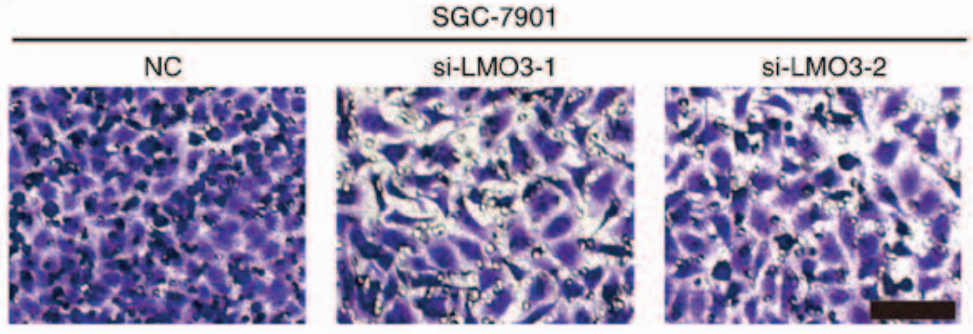

B

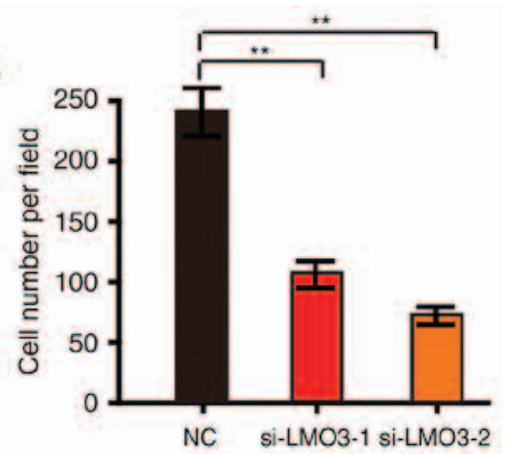

D

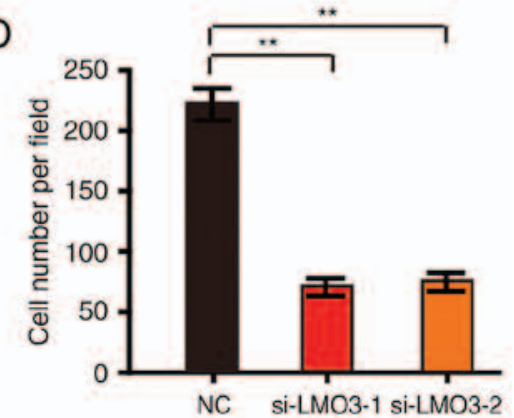

$\mathrm{E}$ MGC-803

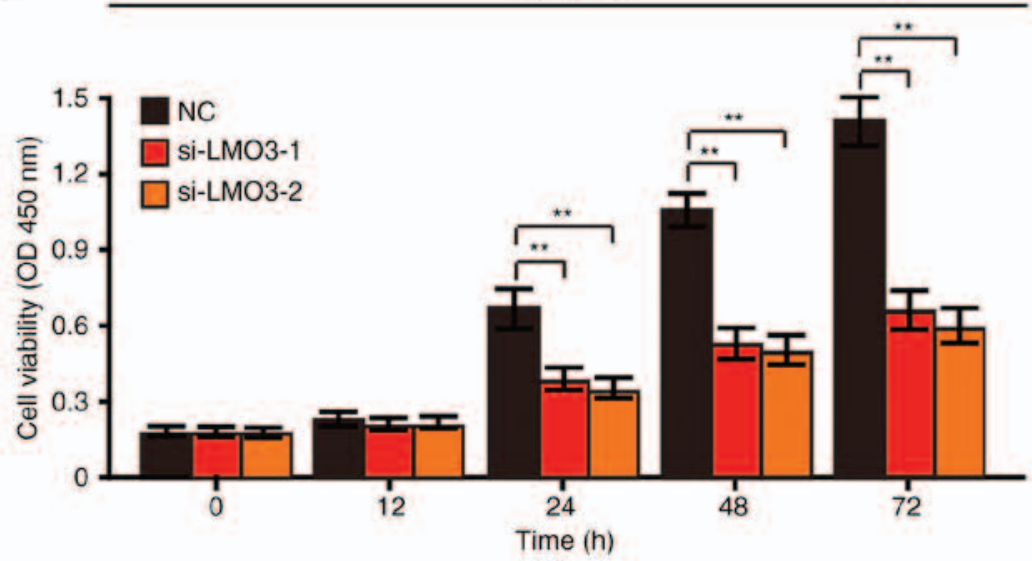

$\mathrm{F}$

SGC-7901

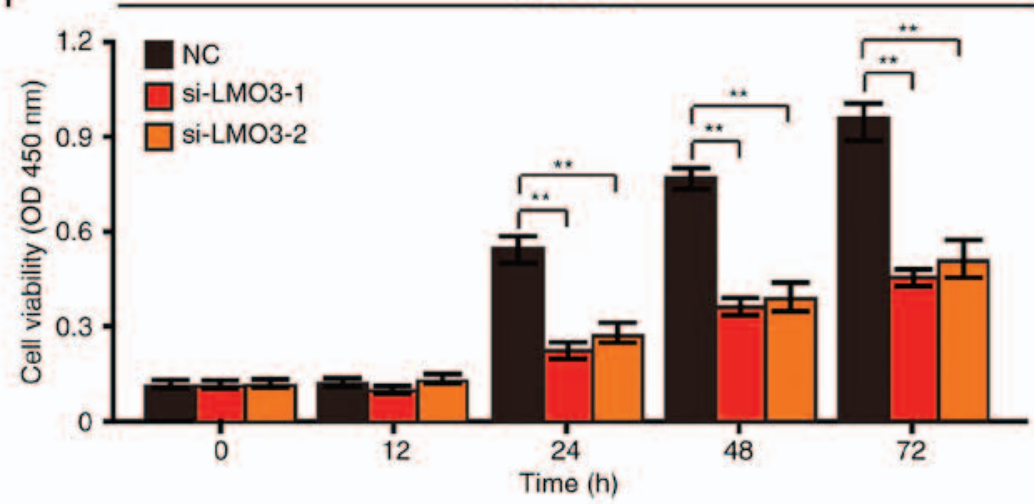

Figure 3. LMO3 knockdown suppresses the invasion and proliferation of gastric cancer cells. (A) Representative photomicrographs of invaded MGC-803 cells transfected with siRNA targeting LMO3 (scale bar, $10 \mu \mathrm{m}$ ) and (B) Quantified numbers of invaded MGC-803 cells in the three groups. (C) Representative photomicrographs of invaded SGC-7901 cells transfected with siRNA targeting LMO3 (scale bar, $10 \mu \mathrm{m}$ ) and (D) Quantified numbers of invaded SGC-7901 cells in the three groups. (E and F) Cell Counting Kit-8 cell viability assay of (E) MGC-803 and (F) SGC-7901 cells transfected with siRNA targeting LMO3 at $0,12,24,48$ and $72 \mathrm{~h}$ time-points. ${ }^{* *} \mathrm{P}<0.01$. OD, optical density; LMO3, LIM domain only 3; NC, negative control; siRNA, small interfering RNA.

GC cell invasion and proliferation were investigated in the presence of dactolisib (inhibitor of mTOR) and CHIR-98014 (inhibitor of GSK3 $\beta$ ). Furthermore, rLMO3 protein was added to BGC-823 and HGC-27 cells, which had low LMO3 
A

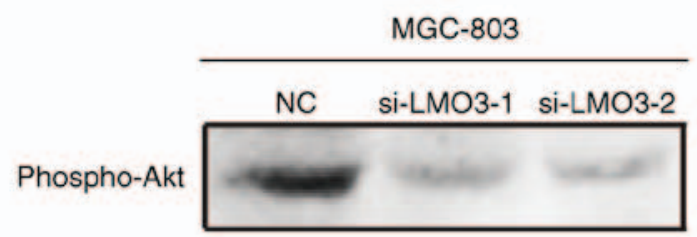

Total-Akt

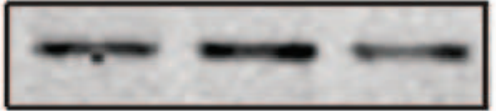

GAPDH

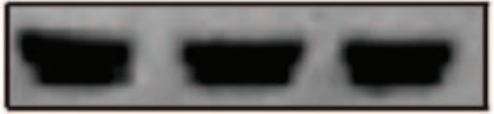

C

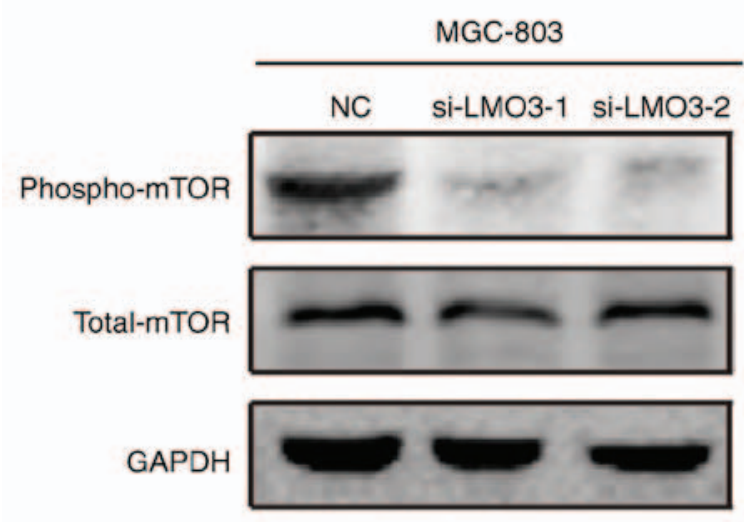

E

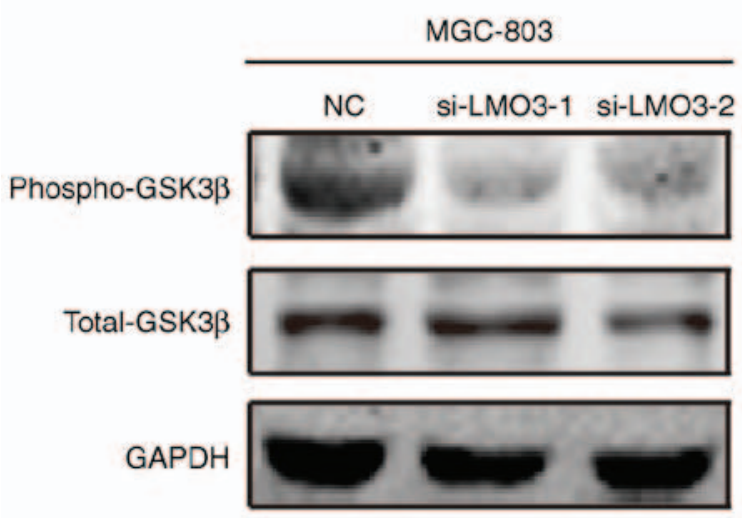

B

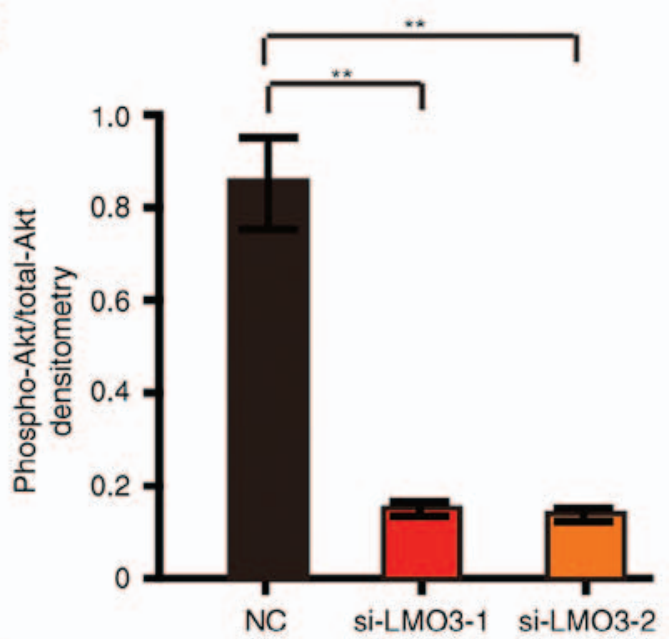

D

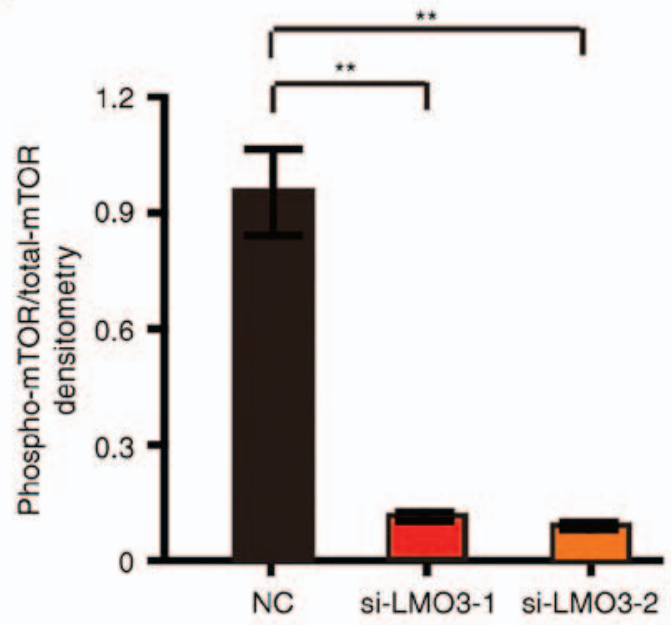

F

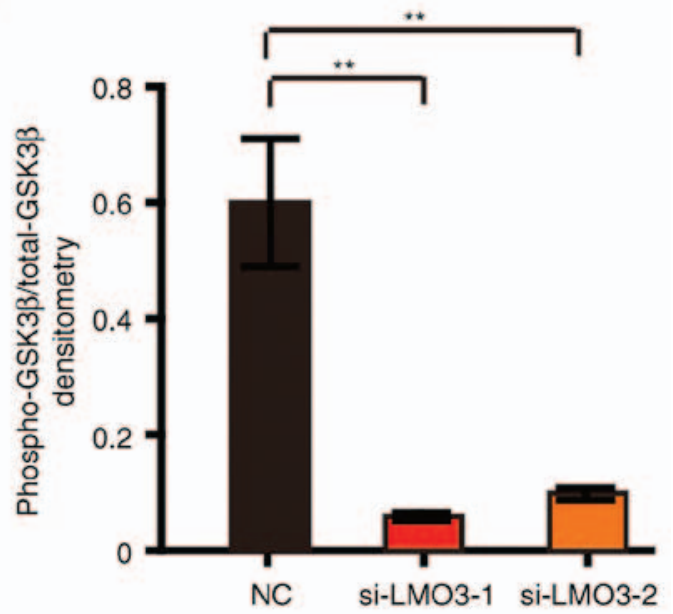

Figure 4. LMO3 knockdown suppresses the phosphorylation of Akt, mTOR and GSK3 $\beta$. (A) Western blot analysis of phospho-Akt and total-Akt in LMO3knockdown and control MGC-803 cells and (B) quantified phospho-Akt/total-Akt ratios. (C) Western blot analysis of phospho-mTOR and total-mTOR in LMO3-knockdown and control MGC-803 cells and (D) quantified phospho-mTOR/total-mTOR ratios. (E) Western blot analysis of phospho-GSK3 $\beta$ and total-GSK3 $\beta$ in LMO3-knockdown and control MGC-803 cells and (F) quantified phospho-GSK3 $\beta /$ total-GSK3 $\beta$ ratios. ${ }^{* *} \mathrm{P}<0.01$. LMO3, LIM domain only 3; NC, negative control; siRNA, small interfering RNA; mTOR, mammalian target of rapamycin; GSK, glycogen synthase kinase.

expression levels, and dactolisib and CHIR-98014 were added after $2 \mathrm{~h}$. It was revealed that dactolisib treatment abrogated rLMO3 protein-induced GC cell invasion (Fig. 5A and B). Furthermore, dactolisib and CHIR-98014 abrogated rLMO3 protein-induced proliferation (Fig. 5C and D).
These results indicated that LMO3-induced GC cell invasion is mainly dependent on Akt/mTOR signaling. Furthermore, LMO3-induced proliferation is mainly dependent on Akt/mTOR and Akt/GSK3 $\beta$ signaling. The signaling cascades are outlined in a schematic in Fig. 5E. 

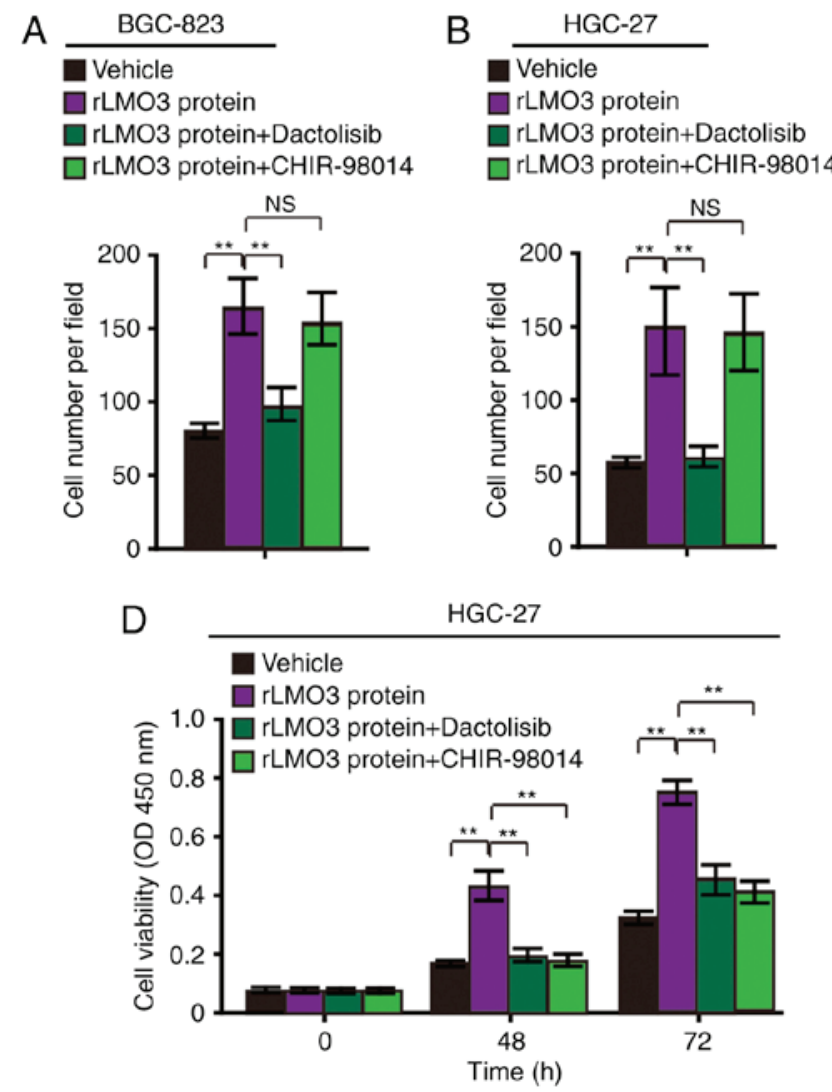
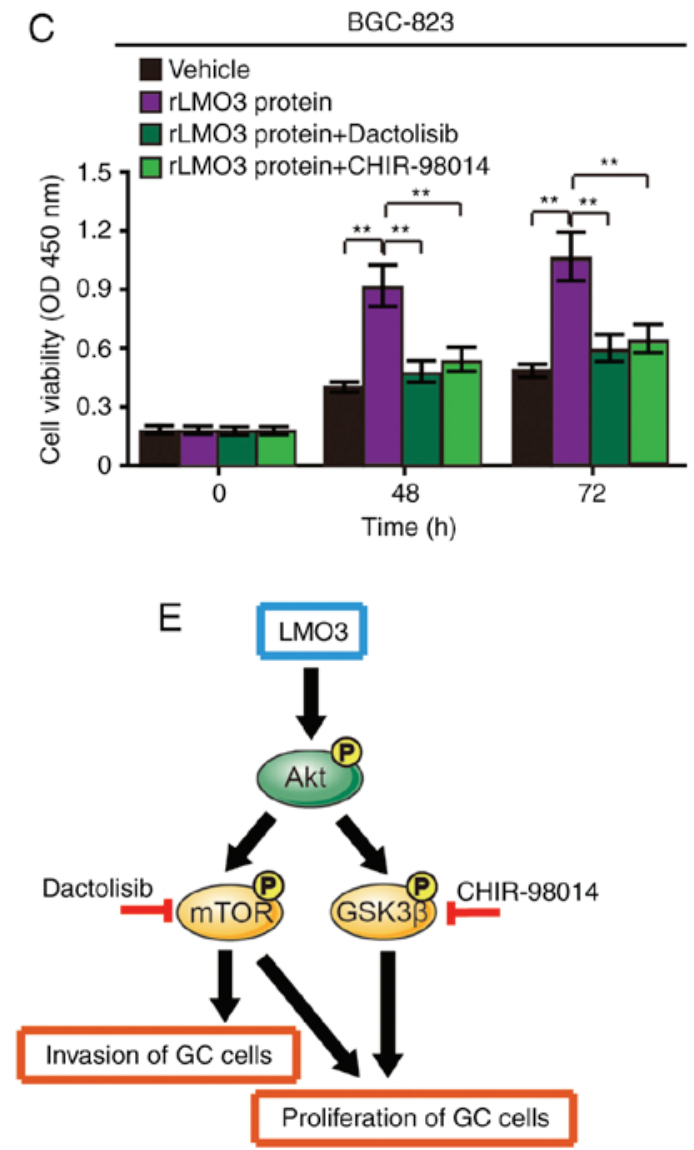

Figure 5. LMO3 promotes GC-cell invasion via Akt-mTOR signaling and promotes GC cell proliferation via Akt-mTOR and Akt-GSK3 $\beta$ signaling. (A) BGC-823 and (B) HGC-27 cells were treated with $50 \mathrm{nM}$ rLMO3 protein, $50 \mathrm{nM}$ rLMO3 protein plus $50 \mathrm{nM}$ Dactolisib (an inhibitor of mTOR), or $50 \mathrm{nM}$ rLMO3 protein plus $50 \mathrm{nM}$ CHIR-98014 (an inhibitor of GSK3ß). Cell invasion was analyzed after $48 \mathrm{~h}$. (C) BGC-823 and (D) and HGC-27 cells were subjected to the abovementioned treatments and cell viability was detected with a Cell Counting Kit- 8 at 0,48 and $72 \mathrm{~h}$ time-points. ${ }^{* *} \mathrm{P}<0.01$. (E) Schematic depicting the mechanism of LMO3-induced GC cell invasion and proliferation. rLMO3, recombinant LIM domain only 3; NC, negative control; siRNA, small interfering RNA; mTOR, mammalian target of rapamycin; GSK, glycogen synthase kinase; GC, gastric cancer; P, phosphate; NS, no significance; OD, optical density.

\section{Discussion}

Numerous studies have assessed LMO3 in various cancer types. It has been reported that LMO3 collaborates with HEN2 to enhance neuroblastoma cell growth $(17,18)$. Notably, microRNA-630 targets LMO3 to regulate lung cancer cell growth and metastasis $(19,20)$. However, the detailed biological functions of LMO3 in GC and the underlying mechanisms have remained to be elucidated. In the present study, the exact roles of LMO3 in GC were deeply investigated for the first time, to the best of our knowledge. By analyzing GC tissue microarray and KMplot datasets, it was identified that the expression of LMO3 was closely associated with the tumor size, differentiation, vascular embolism and TNM stage, as well as poor patient prognoses. These results suggested that LMO3 may have important roles in the development of GC.

We further revealed the biological functions of LMO3 in GC. The invasion and cell viability of GC cells were significantly suppressed by LMO3 knockdown. These results indicated that $\mathrm{LMO} 3$ is involved in the invasion and proliferation of GC cells.

Invasion and metastasis are major concerns during the prognosis and progression of cancer. The Akt/mTOR pathway is pivotal in modulating the invasion and migration of tumor cells $(22,23)$. The present study indicated that knockdown of LMO3 decreases the phosphorylation of Akt and mTOR. By using an inhibitor of mTOR, dactolisib, it was further determined that LMO3-induced GC-cell invasion and proliferation are dependent on Akt-mTOR signaling.

Furthermore, phosphoinositide-3 kinase/Akt/GSK3 $\beta$ signaling has key roles in the regulation of numerous biological functions in various cancer types, including cell migration, invasion, proliferation and apoptosis. The present study identified that LMO3 knockdown decreased the phosphorylation of GSK3 $\beta$, and an inhibitor of GSK3 $\beta$, CHIR-98014, was able to abrogate rLMO3 protein-induced proliferation. Furthermore, the present findings suggested that LMO3-induced GC cell proliferation was dependent on the Akt/GSK3 $\beta$ pathway.

In conclusion, the present study revealed that LMO3 has an important role in GC cell invasion and proliferation. LMO3 promotes GC cell invasion and proliferation through $\mathrm{Akt} / \mathrm{mTOR}$ and Akt/GSK3 $\beta$ signaling. LMO3 may be used as a potential prognostic or therapeutic target for GC in the future.

\section{Competing interests}

The authors declare that they have no competing interests. 


\section{References}

1. Hansson LE, Sparén P and Nyrén O: Survival in stomach cancer is improving: Results of a nationwide population-based Swedish study. Ann Surg 230: 162-169, 1999.

2. Sun X, Mu R, Zhou Y, Dai X, Qiao Y, Zhang S, Huangfu X, Sun J, Li L and Lu F: (1990-1992 mortality of stomach cancer in China). Zhonghua Zhongliu Zazhi 24: 4-8, 2002 (In Chinese)

3. Werner M, Becker KF, Keller G and Höfler H: Gastric adenocarcinoma: Pathomorphology and molecular pathology. J Cancer Res Clin Oncol 127: 207-216, 2001.

4. Becker KF, Keller G and Hoefler H: The use of molecular biology in diagnosis and prognosis of gastric cancer. Surg Oncol 9: 5-11, 2000.

5. Wong SS, Kim KM, Ting JC, Yu K, Fu J, Liu S, Cristescu R, Nebozhyn M, Gong L, Yue YG, et al: Genomic landscape and genetic heterogeneity in gastric adenocarcinoma revealed by whole-genome sequencing. Nat Commun 5: 5477, 2014.

6. Kang G, Hwang WC, Do IG, Wang K, Kang SY, Lee J, Park SH, Park JO, Kang WK, Jang J, et al: Exome sequencing identifies early gastric carcinoma as an early stage of advanced gastric cancer. PLoS One 8: e82770, 2013.

7. Hu B, El Hajj N, Sittler S, Lammert N, Barnes R and MeloniEhrig A: Gastric cancer: Classification, histology and application of molecular pathology. J Gastrointest Oncol 3: 251-261, 2012.

8. Zang ZJ, Cutcutache I, Poon SL, Zhang SL, McPherson JR, Tao J, Rajasegaran V, Heng HL, Deng N, Gan A, et al: Exome sequencing of gastric adenocarcinoma identifies recurrent somatic mutations in cell adhesion and chromatin remodeling genes. Nat Genet 44: 570-574, 2012.

9. Cancer Genome Atlas Research Network. Comprehensive molecular characterization of gastric adenocarcinoma. Nature 513: 202-209, 2014

10. Ding Y, Yang Q, Wang B, Ye G and Tong X: The correlation of MGMT promoter methylation and clinicopathological features in gastric cancer: A systematic review and meta-analysis. PLoS One 11: e0165509, 2016.

11. Baroudi $\mathrm{O}$ and Benammar-Elgaaied $\mathrm{A}$ : Involvement of genetic factors and lifestyle on the occurrence of colorectal and gastric cancer. Crit Rev Oncol Hematol 107: 72-81, 2016.

12. Zhou J, Shen J, Seifer BJ, Jiang S, Wang J, Xiong H, Xie L, Wang $L$ and Sui X: Approaches and genetic determinants in predicting response to neoadjuvant chemotherapy in locally advanced gastric cancer. Oncotarget 8: 30477-30494, 2017.

13. Qi J, Zhang P, Wang Y, Chen H and Li Y: Does total gastrectomy provide better outcomes than distal subtotal gastrectomy for distal gastric cancer? A systematic review and meta-analysis. PLoS One 11: e0165179, 2016.
14. Rabbitts TH: LMO T-cell translocation oncogenes typify genes activated by chromosomal translocations that alter transcription and developmental processes. Genes Dev 12: 2651-2657, 1998

15. Sum EY, Segara D, Duscio B, Bath ML, Field AS, Sutherland RL, Lindeman GJ and Visvader JE: Overexpression of LMO4 induces mammary hyperplasia, promotes cell invasion, and is a predictor of poor outcome in breast cancer. Proc Natl Acad Sci USA 102: 7659-7664, 2005.

16. Visvader JE, Venter D, Hahm K, Santamaria M, Sum EY, O'Reilly L, White D, Williams R, Armes J and Lindeman GJ: The LIM domain gene LMO4 inhibits differentiation of mammary epithelial cells in vitro and is overexpressed in breast cancer. Proc Natl Acad Sci USA 98: 14452-14457, 2001.

17. Aoyama M, Ozaki T, Inuzuka H, Tomotsune D, Hirato J, Okamoto Y, Tokita H, Ohira M and Nakagawara A: LMO3 interacts with neuronal transcription factor, HEN2, and acts as an oncogene in neuroblastoma. Cancer Res 65: 4587-4597, 2005.

18. Isogai E, Ohira M, Ozaki T, Oba S, Nakamura $Y$ and Nakagawara A: Oncogenic LMO3 collaborates with HEN2 to enhance neuroblastoma cell growth through transactivation of Mash1. PLoS One 6: e19297, 2011.

19. Song YF, Hong JF, Liu DL, Lin QA, Lan XP and Lai GX: miR-630 targets LMO3 to regulate cell growth and metastasis in lung cancer. Am J Transl Res 7: 1271-1279, 2015.

20. Watanabe H, Francis JM, Woo MS, Etemad B, Lin W, Fries DF, Peng S, Snyder EL, Tata PR, Izzo F, et al: Integrated cistromic and expression analysis of amplified $N K X 2-1$ in lung adenocarcinoma identifies $L M O 3$ as a functional transcriptional target. Genes Dev 27: 197-210, 2013.

21. Schmittgen TD and Livak KJ: Analyzing real-time PCR data by the comparative C(T) method. Nat Protoc 3: 1101-1108, 2008.

22. Hennessy BT, Smith DL, Ram PT, Lu Y and Mills GB: Exploiting the PI3K/AKT pathway for cancer drug discovery. Nat Rev Drug Discov 4: 988-1004, 2005.

23. Lin HP, Jiang SS and Chuu CP: Caffeic acid phenethyl ester causes p21Cip1 induction, Akt signaling reduction, and growth inhibition in PC-3 human prostate cancer cells. PLoS One 7: e31286, 2012

This work is licensed under a Creative Commons Attribution-NonCommercial-NoDerivatives 4.0 International (CC BY-NC-ND 4.0) License. 